\title{
METODOLOGÍA PARA DETERMINAR Y MEDIR FACTORES QUE INFLUYEN EN LA COMPETITIVIDAD DE PROYECTOS DE SERVICIOS DE EXTENSIÓN AGRARIA
}

\author{
Keneth REATEGUI-DEL AGUILA ${ }^{1}$, Julio ALEGRE${ }^{2}$, Hugo SOPLIN ${ }^{2}$, Manuel CANTO ${ }^{2}$, Silvana \\ VARGAS $^{2}$, Pablo HUERTA ${ }^{3}$ \\ 1 Instituto de Investigaciones de la Amazonia Peruana. Av. José A. Quiñones km. 2.5 Iquitos Perú. \\ Email:kreategui@iiap.org.pe \\ 2 Universidad Nacional Agraria La Molina \\ 3 Comisión Nacional de Ciencia y Tecnología
}

\section{RESUMEN}

Se identificaron y midieron los factores que influyen en la competitividad de 21 proyectos de servicios de extensión ejecutados por el Proyecto INCAGRO en las regiones de Amazonas, San Martín, Loreto y Ucayali. Los factores que explican la variabilidad de la competitividad de los proyectos de servicios de extensión con 83.5\% de probabilidad son: $\mathrm{F} 1$ = APORTES que integra a X1 (meses de duración del proyecto), X2 (aportes de INCAGRO) y X3 (alianza estratégica); F2 = TÉCNICO que agrupa X10 (servicios ofrecidos a clientes) y $\mathrm{X} 11$ (equipos técnicos comprometidos); y el factor F3 = IMPACTO que integra X4 (retorno económico) y X9 (impacto ambiental). Del análisis de regresión lineal múltiple se concluye que la variabilidad de la competitividad de los proyectos de extensión en estudio son explicadas en aproximadamente un $74 \%$ por la ecuación de regresión $\mathrm{Y}=3.429+0.031 \mathrm{~F} 1+0.317 \mathrm{~F} 2+0.107 \mathrm{~F} 3$; en donde el factor F2 TÉCNICO [X10 (servicios ofrecidos a clientes) y X11 (equipos técnicos comprometidos)] tiene el mas alto puntaje y por cada punto que este factor aumente, la competitividad aumentará en promedio en 0.317 , si se mantienen constante los puntajes de los otros factores. Con el factor F3 IMPACTO [X4 (retorno económico) y X9 (impacto ambiental)] se estima que por cada punto adicional de este factor la competitividad aumentará en promedio en 0.107 , manteniéndose constantes los puntajes de los otros factores. Finalmente, por cada punto adicional en el puntaje del Factor F1 APORTES X1 [(meses de duración del proyecto), X2 (aportes de INCAGRO) y X3 (alianza estratégica)], se estima que la competitividad aumentará en promedio solamente 0.031, manteniéndose constantes los puntajes del resto de factores. Con el cálculo de los valores de los coeficientes de correlación no paramétricos de Spearman se concluye que la competitividad de los proyectos de servicios de extensión está significativamente correlacionada con las variables impacto ambientales (X9), servicios ofrecidos a los clientes (X10) y equipos técnicos (X11), es decir, la mejora de los valores de una de estas variables, obviando la presencia de las otras variables permitiría una mejora en el nivel de la competitividad. Los resultados también permiten concluir que la competitividad tiene un grado de asociación débil con las variables X2 (aporte INCAGRO), X3 (aporte alianza estratégica), X4 (retorno económico), X7 (eficacia), X1 (meses), X5 (beneficio costo INCAGRO), X6 (beneficio costo alianza estratégica) y X8 (eficiencia). Todo lo anterior nos permite concluir que para mejorar la competitividad de los proyectos de Servicios de Extensión es imprescindible ofrecer buenos servicios a los clientes, contar con equipos técnicos de extensión comprometidos, buenos retornos económicos de las tecnologías ofrecidas y con bajo impacto ambiental. Las variables tiempo de duración de los proyectos, los aportes económicos y las alianzas estratégicas, tienen un grado de asociación débil con la competitividad de los proyectos de extensión.

PALABRAS CLAVE: Indicadores, variables independientes, competitividad, metodologías. 


\title{
IDENTIFICATION AND MEASUREMENT OF FACTORS AFFECTING COMPETITIVENESS OF AGRICULTURAL EXTENSION PROJECTS IN THE AMAZON
}

\begin{abstract}
Factors influencing the competitiveness of 21 agricultural extension projects were identified and measured. The data used are from a total of 21 extension services projects carried out by Project INCAGRO in the regions of Amazonas, San Martín, Loreto and Ucayali. The factors that explain the variability of the competitiveness with $83.5 \%$ probability are: $\mathrm{F} 1=\mathrm{X} 1$ which integrates CONTRIBUTIONS (month's duration), X2 (contributions INCAGRO) and X3 (strategic alliance); F2 = TECHNICAL grouping X10 (services offered to customers) and X11 (technical teams involved); and F3 = Impact factor that integrates X4 (economic return) and X9 (environmental impact). The multiple linear regression equation $\mathrm{Y}=3.429+\mathrm{F} 2+$ $0.107 \mathrm{~F} 30.031 \mathrm{~F} 10317$, explains the variability of the competitiveness of extension services projects with approximately $74 \%$ of probability; where factor F2 TECHNICAL [X10 (services offered to customers) and $\mathrm{X} 11$ (technical teams involved)] has the highest score and for every point increase, competitiveness will increase on average by 0.317 , if the scores of other factors remain constant. With F3 factor IMPACT [X4 (economic return) and X9 (environmental impact)] is estimated that for every additional point of this factor is estimated to increase competitiveness on average 0.107, remaining constant, the scores of the other factors. Finally, for each additional point in F1 score CONTRIBUTIONS Factor X1 [(month duration of the project), $\mathrm{X} 2$ (contributions INCAGRO) and X3 (strategic alliance)], is estimated to increase competitiveness on average only 0.031 , remaining constant the scores of the other factors. The values of the coefficients of Spearman nonparametric correlation shows that the competitiveness of extension services projects is significantly correlated with environmental impact variables (X9), services offered to customers (X10) and technical equipment (X11), in other words, enhancing the values of these variables, ignoring the presence of other variables allows for an improvement in the level of competitiveness. The results also support the conclusion that the degree of competitiveness has a weak association with the $\mathrm{X} 2$ variables (input INCAGRO), X3 (contributions strategic alliance), X4 (economic return), X7 (effectiveness), X1 (months), $\mathrm{X} 5$ (benefit cost INCAGRO), X6 (benefit-cost strategic alliance) and X8 (efficiency). All this allows us to conclude that to improve the competitiveness of Extension Services projects is essential to provide good services to customers, technical teams of dedicated extension technicians, good economic returns of the technologies offered and low environmental impact. The duration of the project variables, economic contributions, strategic alliances have a weak degree of competitiveness of the partnership extension projects.
\end{abstract}

KEYWORDS: Indicators independent variables, competitiveness, methodologies, extension. 


\section{INTRODUCCIÓN}

El interés y preocupación sobre el tema de la agricultura en la Amazonía se ha incrementado debido al aumento de la población y la baja productividad de los suelos, que afecta a la seguridad alimentaria de la población más vulnerable en esta región (Janvry, 2009). Al mismo tiempo, la producción y en especial la exportación de productos del agro amazónico peruano han tenido un notable crecimiento debido a su adaptación a las exigencias de los mercados agro-alimenticios mundiales (Benites, 2013). Cualquier iniciativa que busque impulsar la investigación agraria en la Amazonia tendrá que considerar modalidades de trabajo que estén de acuerdo a las particularidades biofísicas de los ecosistemas amazónicos y aspectos socioculturales de la región, y también a la generación de bienestar social para la población.

Los servicios de extensión son servicios profesionales orientados a atender la demanda por asistencia técnica y capacitación, permitiendo aplicar conocimientos especializados disponibles para resolver problemas en la producción, posproducción o poscosecha, procesamiento o transformación y comercialización de los productos agropecuarios y forestales (Norton, 2004). También forman parte de estos servicios, los orientados a mejorar la gestión de las unidades productivas, así como de las organizaciones de productores. Los datos empleados provienen de un total de 21 proyectos de servicios a la extensión cofinanciados por el Programa de Innovación y Competitividad del Agro (INCAGRO) en el ámbito de la Unidad Descentralizada VII que comprende las regiones de Amazonas, San Martín, Loreto y Ucayali, ejecutados desde el año 2001 hasta el 2010. Los costos de la capacitación por competencias de los agentes de extensión y de los operadores de servicios era cofinanciada por INCAGRO. Para la aprobación de los proyectos de extensión se necesitó información adecuada y confiable, razón por la cual se hace necesario métodos estadísticos que ayuden a cuantificar, analizar y explicar resultados de las interacciones de variables dependientes e independientes, de proyectos ejecutados $\mathrm{y} / \mathrm{o}$ en desarrollo (Benites y Wiener, 1998).

En los proyectos de extensión, la dependencia de la competitividad ante otras variables independientes, tales como: aporte económico y tiempo, sensibilidad económica, impacto de eficiencia, impacto socio ambiental es determinante para obtener los objetivos esperados. Ortiz (2009), sostiene que un nuevo y moderno enfoque de extensión hace necesario revisar también el marco teórico del cual forma parte. Hoy en día los "servicios de extensión" pueden ser definidos como un "conjunto de organismos y entidades que apoyan a personas involucradas en la producción agraria para resolver problemas y para obtener información, desarrollar habilidades y conocer tecnologías para mejorar sus medios de vida y su bienestar". En los proyectos de servicios de extensión, la competitividad es la variable determinante para obtener los objetivos esperados. Sin embargo, se necesita cuantificar la dependencia de la competitividad ante otras variables independientes, tales como: aportes económicos y tiempo, impacto sensibilidad económica, impacto eficiencia, impacto socio ambiental, etc. El estudio busca dar sustento científico a la toma de decisiones estratégicas técnicas y políticas para proponer proyectos de extensión y garantizar su eficiencia y éxito. La competitividad es la variable dependiente y determinante para disminuir la pobreza. Por último, las variables independientes que determinan la competitividad, deberían ser consolidadas e implementadas en políticas gubernamentales.

Norton (2004) ofrece varias propuestas para la extensión agrícola: (a) los servicios de extensión deben orientarse más hacia los clientes y hacia la rendición de cuentas a los clientes, que son los agricultores; (b) la descentralización de los servicios de extensión pública probablemente mejorará su eficacia, ya que los lleva más cerca de sus clientes, los productores; (c) la extensión debería facilitar no sólo la adquisición de destrezas agrícolas, sino también habilidades en la administración de las fincas, contabilidad, comercialización, lazos con instituciones de crédito $\mathrm{y}$ proveedores de insumos y organizaciones comunales; (d) es necesario explorar diferentes formas de financiación de la extensión, incluyendo la que permite compartir los costos con los productores que pueden permitírselo. La contribución financiera de los productores los pone en mejor posición para juzgar la calidad del servicio y dirigirlo hacia sus propias prioridades.

\section{MATERIAL Y MÉTODOS}

El estudio comprende 21 proyectos de servicios de extensión ejecutados por el Programa para la Innovación y Competitividad del Agro Peruano PIEA-INCAGRO en el ámbito de la Unidad Descentralizada VII que comprende las regiones de Amazonas, San Martín, Loreto y Ucayali. En la tabla 1 se presentan los 21 proyectos de servicios de extensión a evaluarse: seis de café, cuatro de vacunos, dos de peces; y uno de piña, seda, cacao, palma, miel, chancaca, forraje, cuyes y avestruz. 
Esto servirá para hacer los análisis descriptivos correspondientes para negar o afirmar la hipótesis del estudio que la competitividad de los proyectos de Servicios de Extensión depende de una o más de las siguientes variables: X1 meses (tiempo de desarrollo del proyecto); X2 aporte INCAGRO (monto total aportado por INCAGRO); X3 aporte alianza estratégica (monto aportado por los socios que presentan la iniciativa); X4 retorno económico (retorno económico calculado en base al plan de negocios post proyecto); X5 beneficio costo INCAGRO [cálculo estimado en base a la relación retorno económico total (X4) y el aporte de INCAGRO (X2)]; X6 beneficio costo alianza estratégica [(cálculo estimado en base a la relación retorno económico total X4 y el aporte de la alianza estratégica (X3)]; X7 eficacia (indicador de retorno económico, a menor tiempo mayor retorno); X8 eficiencia (indicador de retorno económico; a menor tiempo y menor costo, mayor retorno); X9 impacto ambiental (calificación desarrollada considerando la base de datos de INCAGRO); X10 servicios a clientes (servicios ofrecidos durante la ejecución del proyecto); X11 equipos técnicos comprometidos (calificación desarrollada en base a la calidad de aporte del equipo técnico ejecutor del proyecto).

\section{Análisis estadístico}

Se aplicó la técnica denominada análisis factorial multivariado para reducir la dimensionalidad de los datos. Seguidamente se usó la regresión múltiple para identificar y medir variables que influyen en la competitividad de los proyectos de investigación y de esta forma establecer un modelo que permita obtener predicciones acerca de su competitividad. Finalmente, se midió el grado de asociación entre la competitividad y las variables independientes con el cálculo y la verificación de la significación estadística de los coeficientes de correlación no paramétrica de Spearman.

\section{RESULTADOS}

\section{Análisis factorial para reducir la dimensionalidad de los datos}

De las 11 variables inicialmente identificadas se seleccionaron siete variables: X1 (Meses duración del proyecto), X2 (Aporte INCAGRO), X3 (Aporte alianza estratégica), X4 (Retorno económico), X9 (Impacto ambiental), X10 (Servicios a clientes), $\mathrm{X} 11$ (Equipos técnicos comprometidos). Con estas variables se formaron grupos homogéneos procurando que unos grupos sean independientes de otros usando el análisis de la matriz de correlaciones.
La determinante de la matriz encontrada es cercana a cero, lo que indica la presencia de correlaciones importantes entre las variables involucradas y la conformidad del análisis factorial. Además, se puede observar que ya no se presentan variables lineales y matemáticamente dependientes de otras variables, lo que permitirá obtener la matriz inversa de correlaciones, que es la base para el cálculo de las comunalidades iniciales y para el cálculo de la matriz anti imagen.

Como se puede apreciar se ha obtenido la inversa de la matriz de correlaciones, debido a que esta es una matriz definida positiva.

Como puede observarse en la tabla 4, la matriz anti-imagen de correlaciones obtenida no difiere de la matriz identidad. Para comprobar este resultado se calculó el índice de adecuación muestral de Kaiser Meyer - Olkin (KMO) y se realizó la prueba de esfericidad de Bartlett. El valor de KMO mayor a 0.5 indica que es adecuado el modelo factorial resultante, también de manera concordante la prueba de esfericidad de Bartlett resultó significativa, lo que representa que la matriz de correlaciones es diferente a la matriz identidad, validando el análisis factorial.

\section{Extracción de factores y las variables que las conforman}

La extracción de factores, basada en la agrupación de variables en factores o componentes que resulten no independientes entre sí y que a su vez internamente estén constituidas por la variable de mayor correlación, fue efectuada por el análisis de comunalidades.

La tabla 6 muestra que la variable peor explicada por el modelo es la X1 (meses), donde sólo el 66.9\% de su variabilidad es explicada por el factorial encontrado; mientras que la variable X3 (Aporte de la Alianza estratégica) es la mejor explicada por el modelo, ya que el porcentaje de explicación por el modelo factorial encontrado alcanza el 91\% de su variabilidad. Al respecto, se puede afirmar que la duración de los proyectos responde a un plan determinado, es decir, están previamente establecidos, por lo que es de esperar que su variablidad no esté asociada a otras variables aleatorias cuyos valores están más influenciados por la marcha propia del desarrollo del proyecto. La variabilidad total de los datos se muestra en la tabla 7.

La tabla 7 muestra que con 3 factores se alcanza una explicación del $83.460 \%$ de la variabilidad total de los datos. Asimismo, se muestra este porcentaje considerando los diferentes números de posibles factores para el modelo elegido. Las figura 1 ilustra 


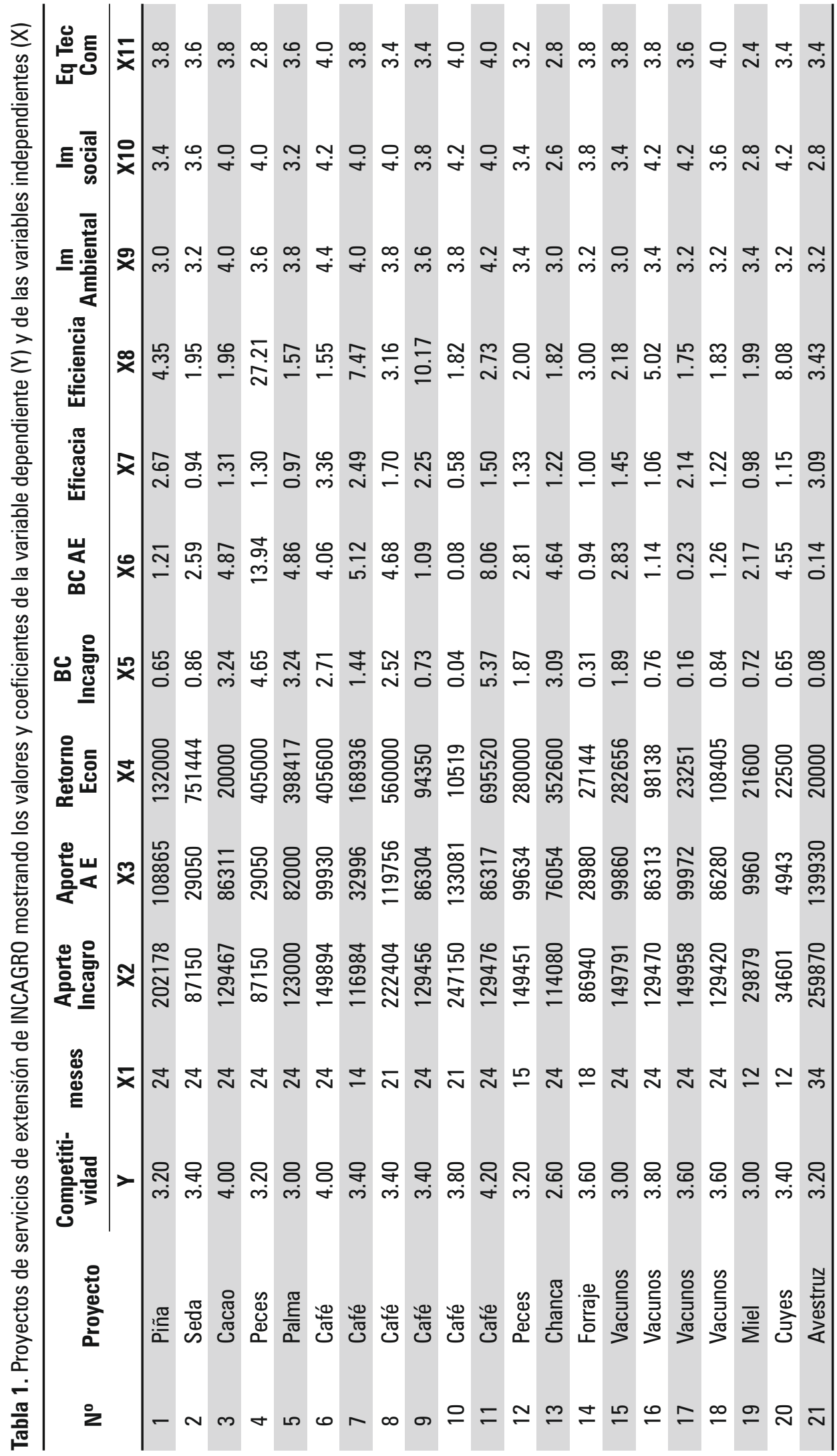


que hasta con tres factores como mínimo se tienen autovalores mayores a uno. Aquí podríamos decir que con los tres primeros factores se explican el $83.460 \%$ del total de la variabilidad, por ello se considera conveniente agruparlos en tres factores.

Observamos que el punto de inflexión se da cuando el número de componentes es igual a tres, por eso escogemos ese número de componentes, y eso se puede visualizar mejor en la matriz de componentes. La matriz de componentes o la de componentes rotados, muestra las correlaciones entre las variables y los componentes, es decir, la estructura factorial que indica las variables que integran cada factor. Para determinar este resultado se escoge para cada variable la correlación más alta con los componentes.

En la tabla 8 se observa que hay tres factores: F1 $=$ APORTES que agrupa a X1 (meses de duración del proyecto), X2 (aportes de INCAGRO) y X3 (alianza estratégica) constituyen en efecto un conjunto de variables que pueden incidir significativamente sobre la competitividad; F2 = TÉCNICO que agrupa X10 (servicios al cliente) y X11 (equipos técnicos comprometidos) es también un posible factor que incide sobre la competitividad; y F3 = IMPACTO que integra X4 (retorno económico) y X9 (impacto ambiental) también puede constituirse en un factor que influya significativamente sobre la competitividad.

El análisis factorial permitió reducir la dimensionalidad del problema de las 11 variables independientes iniciales como los posibles factores explicativos de la competitividad, de los proyectos de servicios de extensión. Obtenidos los factores y sus variables, se procedió a obtener las puntuaciones factoriales (Tabla 9) conjuntamente con los valores de la variable dependiente competitividad Y.

\section{Análisis de regresión lineal múltiple}

Con los datos de la tabla 9 se realizó un análisis de regresión lineal. Antes de estimar el modelo de regresión de la variable la competitividad en los proyectos de extensión en función de los puntajes factoriales, se procedió a verificar la normalidad de la variable dependiente mediante la prueba de Kolmogorov-Smirnov que permite medir el grado de concordancia existente entre la distribución de un conjunto de datos y una distribución teórica específica. Su objetivo es señalar si los datos provienen de una población que tiene la distribución teórica especificada, en este caso la distribución Normal.

El valor de 0.746 de la significación asintótica permite concluir que la distribución de la variable competitividad se ajusta a una distribución Normal. Probada la Normalidad, se procedió a realizar el análisis de regresión, los siguientes son los resultados:

Se encontró que la competitividad de los proyectos de extensión es explicada en aproximadamente un $73.8 \%$ por los puntajes factoriales.

En la tabla 12 se observa una regresión altamente significativa entre la competitividad de los proyectos de extensión y los puntajes factoriales, es decir la competitividad depende de manera significativa de al menos uno de los puntajes factoriales obtenidas.

El modelo de regresión estimado es: $\mathrm{Y}=3.429+$ $0.031 \mathrm{~F} 1+0.317 \mathrm{~F} 2+0.107 \mathrm{~F} 3$; en donde el factor F2 TÉCNICO [X10 (servicios ofrecidos a clientes) y $\mathrm{X} 11$ (equipos técnicos comprometidos)] tiene el mas alto puntaje y por cada punto que este factor aumente la competitividad aumentará en promedio en 0.317 , si se mantienen constante los puntajes de los otros factores. Con el factor F3 IMPACTO [X4 (retorno económico) y X9 (impacto ambiental)] se estima que por cada punto adicional de este factor se estima que la competitividad aumentará en promedio en 0.107 , manteniéndose constante, los puntajes de los otros factores. Finalmente, por cada punto adicional en el puntaje del Factor F1 APORTES X1 [(meses de duración del proyecto), X2 (aportes de INCAGRO) y X3 (alianza estratégica)], se estima que la competitividad aumentará en promedio solamente 0.031, manteniéndose constante, los puntajes de los otros factores.

También se ha encontrado, de acuerdo a la significancia de la prueba de $\mathrm{t}$, a un nivel de significación del 5\% que la competitividad, depende significativamente del factor Técnico (F2), del factor Aportes (F3) y la dependencia no es significativa del factor Aportes (F1). Estos últimos resultados significan que para la explicación de la competitividad de los proyectos de Servicios de extensión se debe prestar mucha atención a las variables que constituyen los factores 2 y 3 , es decir mejorar calificaciones respectos a estas variables, adoptando las medidas que conlleven a este fin.

\section{Correlación no paramétrica de Spearman}

En la tabla 14 se presenta el grado de asociación no paramétrica entre la competitividad y las 11 variables independientes originalmente consideradas.

Los resultados obtenidos indican la competitividad de los proyectos de extensión está significativamente correlacionada de manera directa 
con las variables Impacto Ambiental (X9), Impacto Social (X10) y Equipos Técnicos (X11), es decir, la mejora de los valores de una de estas variables, obviando la presencia de las otras variables permitiría una mejora en el nivel de la competitividad. Los resultados también muestran que la competitividad tiene un grado de asociación débil con las variables X2 (Aporte INCAGRO), X3 (Aporte alianza estratégica), X4 (Retorno económico), X7 (Eficacia), X1 (Meses), X5 (Beneficio costo INCAGRO), X6 (Beneficio costo alianza estratégica) y X8 (Eficiencia).

Tabla 2. Matriz de correlaciones ${ }^{a}$

\begin{tabular}{|c|c|c|c|c|c|c|c|c|}
\hline & & $\mathrm{X} 1$ & $\mathrm{X} 2$ & X3 & $\mathrm{X} 4$ & X9 & $\mathrm{X} 10$ & $\mathrm{X} 11$ \\
\hline & $\mathrm{X} 1$ & 1,000 & ,551 & ,630 & , 165 &,- 049 &,- 157 & 2,267 \\
\hline & $\mathrm{X} 2$ &, 551 & 1,000 & ,928 & ,056 & 087 &,- 009 & ,421 \\
\hline & X3 & ,630 & ,928 & 1,000 & , 193 & 109 &,- 026 & ,398 \\
\hline \multirow[t]{7}{*}{ Correlación } & $\mathrm{X} 4$ & , 165 & ,056 & ,193 & , 1000 &, 573 &, 088 &, 052 \\
\hline & X9 &,- 049 & ,087 & 109 &, 573 & 1,000 & ,483 & ,300 \\
\hline & $\mathrm{X} 10$ &,- 157 &,- 009 &,- 026 & ,088 & ,483 & 1,000 &, 547 \\
\hline & $\mathrm{X} 11$ & 267 & ,421 & ,398 & ,052 & ,300 &, 547 & 1,000 \\
\hline & $\mathrm{X} 1$ & & ,005 &, 001 & ,237 & ,416 & ,248 & 121 \\
\hline & $\mathrm{X} 2$ & ,005 & & 000 & ,405 & ,354 & ,484 & ,029 \\
\hline & X3 & ,001 &, 000 & & ,202 & ,319 &, 455 &, 037 \\
\hline \multirow[t]{4}{*}{ Sig. (Unilateral) } & $\mathrm{X} 4$ & 237 & ,405 & 202 & & ,003 & ,353 & ,412 \\
\hline & $\mathrm{X} 9$ & ,416 & ,354 & ,319 & ,003 & &, 013 &, 094 \\
\hline & $\mathrm{X} 10$ & 248 & ,484 & ,455 & ,353 & ,013 & & ,005 \\
\hline & $\mathrm{X} 11$ & 121 &, 029 &, 037 & , 412 & ,094 &, 005 & \\
\hline
\end{tabular}

a. Determinante $=.015$

Tabla 3. Inversa de la matriz de correlaciones

\begin{tabular}{rrrrrrrr}
\hline & X1 & X2 & X3 & X4 & X9 & X10 & X11 \\
\hline X1 & 1,845 &, 366 & $-1,322$ &,- 250 &, 293 &, 352 &,- 388 \\
X2 &, 366 & 8,701 & $-8,263$ & 1,437 &,- 700 &, 453 &,- 585 \\
X3 & $-1,322$ & $-8,263$ & 9,757 &,- 1473 &, 499 &,- 101 &,- 070 \\
X4 &,- 250 & 1,437 & $-1,437$ & 1,946 & $-1,283$ &, 290 &, 172 \\
X9 &, 293 &,- 700 &,- 700 & $-1,283$ & 2,192 &,- 829 &,- 118 \\
X10 &, 352 &, 453 &, 453 &, 290 &,- 829 & 2,053 & $-1,135$ \\
X11 &,- 388 &,- 585 &,- 585 &, 172 &,- 118 & $-1,135$ & 2,026 \\
\hline
\end{tabular}


FOLIA

Amazónica

Tabla 4. Matriz de anti-imagen ${ }^{\mathrm{a}}$

\begin{tabular}{|c|c|c|c|c|c|c|c|c|}
\hline & & $\mathrm{X} 1$ & $\mathrm{X} 2$ & $\mathrm{X} 3$ & $X 4$ & X9 & X10 & X11 \\
\hline & $\mathrm{X} 1$ &, 542 & ,023 &,- 073 &,- 070 &,- 072 & ,093 &,- 104 \\
\hline & $X 2$ & ,023 & ,115 &,- 097 & ,085 &,- 037 & ,025 &,- 033 \\
\hline & X3 &,- 073 &,- 097 & 102 &,- 078 & ,023 &,- 005 &,- 004 \\
\hline \multirow[t]{7}{*}{ Covarianza anti-imagen } & $X 4$ &,- 070 & ,085 &,- 078 &, 514 &,- 301 & ,073 &, 044 \\
\hline & X9 & ,072 &,- 037 & ,023 &,- 301 & ,456 & 184 &,- 027 \\
\hline & $\mathrm{X} 10$ & ,093 & ,025 &,- 005 & ,073 & -184 & 487 &,- 273 \\
\hline & $\mathrm{X} 11$ & -104 &,- 033 &,- 004 & ,044 &,- 027 &,- 273 & ,494 \\
\hline & $\mathrm{X} 1$ &, $792^{\mathrm{a}}$ & ,091 &,- 312 & -,132 & , 146 & 181 &,- 201 \\
\hline & $X 2$ & ,091 &, $577^{\mathrm{a}}$ & -897 & ,349 &,- 160 & 107 & -139 \\
\hline & X3 &,- 312 &,- 897 &, $588^{a}$ &,- 338 & , 108 &,- 023 &,- 016 \\
\hline \multirow[t]{4}{*}{ Correlación anti-imagen } & $\mathrm{X} 4$ &,- 132 & ,349 &,- 338 & $378^{\mathrm{a}}$ &,- 621 & 145 & ,087 \\
\hline & $\mathrm{Xg}$ & 146 &,- 160 & 108 &,- 621 &, $529^{\mathrm{a}}$ &,- 391 &,- 056 \\
\hline & $\mathrm{X} 10$ & 181 & 107 &,- 023 & , 145 &,- 391 &, $517^{\mathrm{a}}$ &,- 557 \\
\hline & $\mathrm{X} 11$ &,- 201 &,- 139 &,- 016 & , 087 &,- 056 &,- 557 & $678^{\circ}$ \\
\hline
\end{tabular}

a. Medida de adecuación muestral

Tabla 5. KMO y prueba de Bartlett

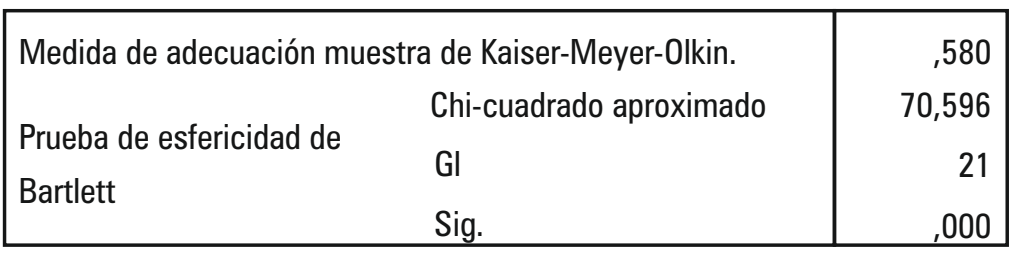

Tabla 6. Comunalidades

\begin{tabular}{lcc}
\hline & Inicial & Extracción \\
\hline X1 & 1,000 &, 669 \\
X2 & 1,000 &, 865 \\
X3 & 1,000 &, 910 \\
X4 & 1,000 &, 893 \\
X9 & 1,000 &, 834 \\
X10 & 1,000 &, 860 \\
X11 & 1,000 &, 811 \\
\hline
\end{tabular}


Tabla 7. Varianza total explicada

\begin{tabular}{|c|c|c|c|c|c|c|c|c|c|}
\hline \multirow{2}{*}{$\begin{array}{l}\text { Compo- } \\
\text { nente }\end{array}$} & \multicolumn{3}{|c|}{ Autovalores iniciales } & \multicolumn{3}{|c|}{$\begin{array}{l}\text { Sumas de las saturaciones al } \\
\text { cuadrado de la extracción }\end{array}$} & \multicolumn{3}{|c|}{$\begin{array}{l}\text { Sumas de las saturaciones a } \\
\text { cuadrado de la rotación }\end{array}$} \\
\hline & Total & $\begin{array}{l}\% \text { de la } \\
\text { varianza }\end{array}$ & $\begin{array}{c}\% \\
\text { acumulado }\end{array}$ & Total & $\begin{array}{l}\% \text { de la } \\
\text { varianza }\end{array}$ & $\begin{array}{c}\% \\
\text { acumulado }\end{array}$ & Total & $\begin{array}{l}\% \text { de la } \\
\text { varianza }\end{array}$ & $\begin{array}{c}\% \\
\text { acumulado }\end{array}$ \\
\hline 1 & 2,775 & 39,642 & 39,642 & 2,775 & 39,642 & 39,642 & 2,580 & 36,863 & 36,863 \\
\hline 2 & 1,883 & 26,894 & 66,535 & 1,883 & 26,894 & 66,535 & 1,717 & 24,531 & 61,394 \\
\hline 3 & 1,185 & 16,925 & 83,460 & 1,185 & 16,925 & 83,460 & 1,545 & 22,066 & 83,460 \\
\hline 4 &, 521 & 7,447 & 90,907 & & & & & & \\
\hline 5 & ,310 & 4,431 & 95,338 & & & & & & \\
\hline 6 & ,271 & 3,866 & 99,204 & & & & & & \\
\hline 7 & ,056 & ,796 & 100,000 & & & & & & \\
\hline
\end{tabular}

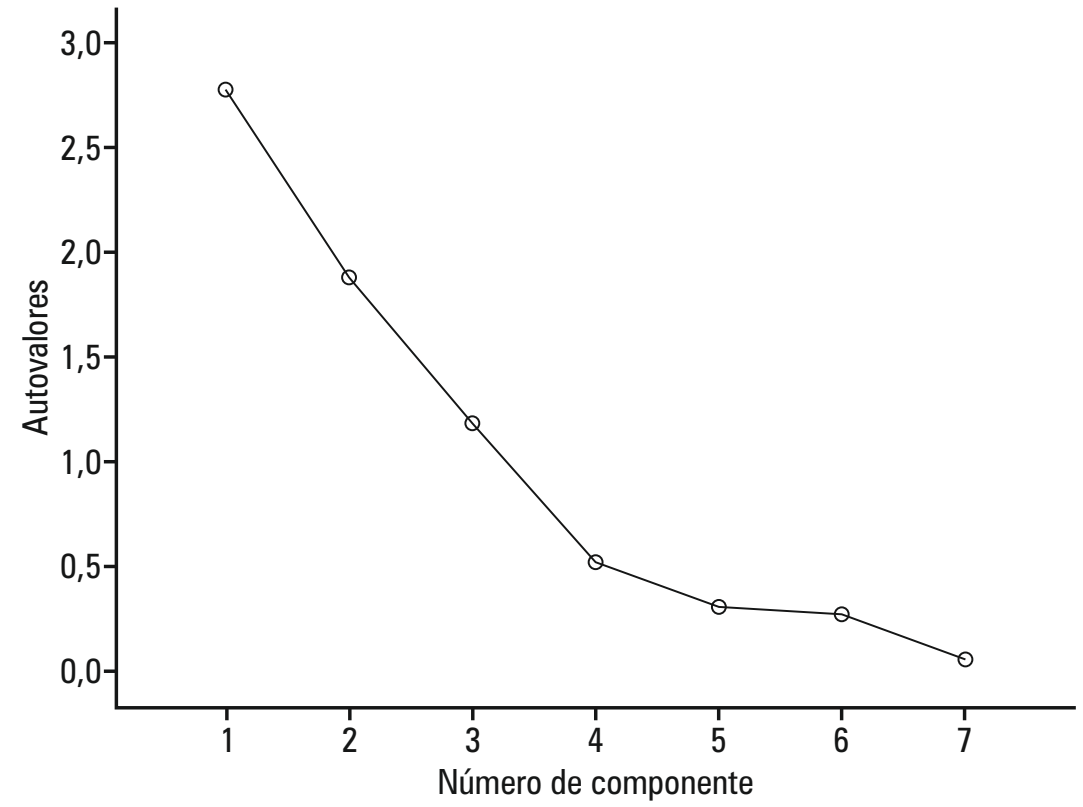

Figura 1. Gráfico de sedimentación 
Tabla 8. Matriz de componentes rotados ${ }^{\mathrm{a}}$

\begin{tabular}{lccc}
\hline & \multicolumn{3}{c}{ Componente } \\
\cline { 2 - 4 } X1 & $\mathbf{1}$ & $\mathbf{2}$ & $\mathbf{3}$ \\
\hline X2 &, 808 &,- 115 &, 061 \\
X3 &, 914 &, 168 &,- 016 \\
X4 &, 943 &, 106 &, 099 \\
X9 &, 126 &,- 080 &, 933 \\
X10 &,- 026 &, 450 &, 794 \\
X11 &,- 164 &, 897 &, 171 \\
\hline
\end{tabular}

a La rotación ha convergido en 5 iteraciones.

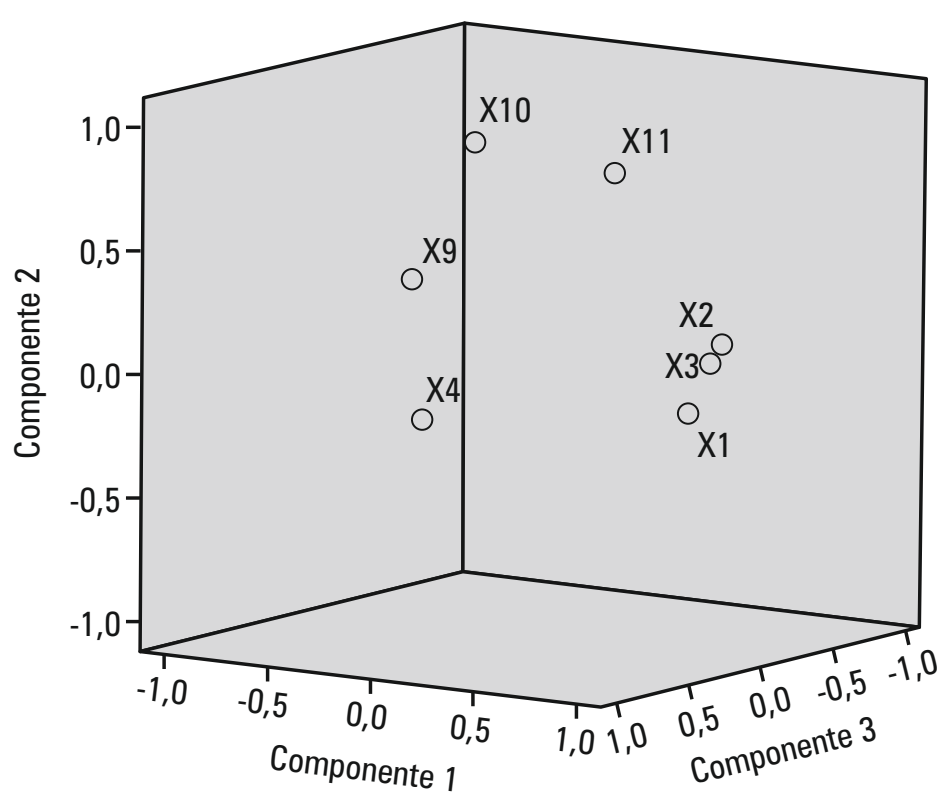

Figura 2. Gráfico de componentes en espacio rotado 
Tabla 9. Matriz de anti-imagen ${ }^{\text {a }}$

\begin{tabular}{|c|c|c|c|c|}
\hline PROYECTO & APORTES (FACT1) & TÉCNICO (FACT2) & IMPACTO (FACT3) & $\mathbf{Y}$ \\
\hline 1 & 1,02452 &,- 12807 &,- 98897 & 3,20 \\
\hline 2 &,- 52196 &,- 07057 &,- 79512 & 3,40 \\
\hline 3 & 11846 & ,53298 & 1,15104 & 4,00 \\
\hline 4 & $-1,01483$ &,- 75836 & 1,03870 & 3,20 \\
\hline 5 & 22518 & -62204 & ,96863 & 3,00 \\
\hline 6 & ,29666 & 1,15216 & 1,47085 & 4,00 \\
\hline 7 & $-1,11676$ & 1,07408 & , 24518 & 3,40 \\
\hline 8 & ,72894 & ,00943 & 1,40661 & 3,40 \\
\hline 9 & ,09405 & ,07262 & -22789 & 3,40 \\
\hline 10 & 1,02224 & 1,50998 & -63291 & 3,80 \\
\hline 11 & 17040 & ,53142 & 2,21490 & 4,20 \\
\hline 12 &,- 13123 & -61235 & 16043 & 3,20 \\
\hline 13 & , 19688 & $-2,42383$ & 21019 & 2,60 \\
\hline 14 & -91432 &, 58264 & $-1,07544$ & 3,60 \\
\hline 15 & ,65570 &,- 33253 &,- 44096 & 3,00 \\
\hline 16 & 12101 & ,88461 & -62617 & 3,80 \\
\hline 17 & ,34066 & 67681 & $-1,03348$ & 3,60 \\
\hline 18 & ,35572 & ,35915 & -84389 & 3,60 \\
\hline 19 & $-1,92243$ & $-1,87825$ &,- 30382 & 3,00 \\
\hline 20 & $-2,02342$ & ,70227 &,- 96924 & 3,40 \\
\hline 21 & 2,29452 & $-1,26214$ &,- 92862 & 3,20 \\
\hline
\end{tabular}

Tabla 10. Prueba de Kolmogorov-Smirnov para una muestra

\begin{tabular}{|ll|r|}
\hline & & \multicolumn{1}{|c|}{$\mathrm{Y}$} \\
\hline $\mathrm{N}$ & Media & 21 \\
Parámetros normalesa,b & Desviación típica & 3,4286 \\
& Absoluta &, 39133 \\
& Positiva &, 148 \\
Diferencias más extremas &, 148 \\
& Negativa &,- 090 \\
Z de Kolmogorov-Smirnov & &, 679 \\
Sig. asintót (bilateral) & &, 746 \\
\hline
\end{tabular}

Tabla 11. Resumen del modelo

\begin{tabular}{ccccc}
\hline Modelo & $\mathbf{R}$ & R cuadrado & $\begin{array}{c}\text { R cuadrado } \\
\text { corregida }\end{array}$ & $\begin{array}{c}\text { Error típ. de la } \\
\text { estimación }\end{array}$ \\
\hline 1 &, $859^{\mathrm{a}}$ &, 738 &, 691 &, 21747 \\
\hline
\end{tabular}


Tabla 12. ANOVA

\begin{tabular}{ccccccc}
\hline \multicolumn{2}{c}{ Modelo } & $\begin{array}{c}\text { Suma de } \\
\text { cuadrados }\end{array}$ & gl & $\begin{array}{c}\text { Media } \\
\text { cuadrática }\end{array}$ & F & Sig. \\
\hline & Regresión & 2,259 & 3 &, 753 & 15,922 &, $000^{\mathrm{b}}$ \\
\hline & Residual &, 804 & 17 &, 047 & & \\
\hline & Total & 3,063 & 20 & & & \\
\hline
\end{tabular}

a. Variable dependiente: $Y$; b. variables predictoras.

Tabla 13. Coeficientes de regresión ${ }^{a}$

\begin{tabular}{|c|c|c|c|c|c|c|c|}
\hline & \multirow{2}{*}{\multicolumn{2}{|c|}{ Modelo }} & \multicolumn{2}{|c|}{$\begin{array}{c}\text { Coeficientes } \\
\text { no estandarizados }\end{array}$} & \multirow{2}{*}{$\begin{array}{c}\begin{array}{c}\text { Coeficientes } \\
\text { tipificados }\end{array} \\
\text { Beta }\end{array}$} & \multirow[t]{2}{*}{$\mathbf{t}$} & \multirow[t]{2}{*}{ Sig } \\
\hline & & & B & Error típ. & & & \\
\hline & (Constante) & & 3,429 & ,047 & & 72,249 &, 000 \\
\hline & REGR factor score & 1 for analysis 1 & ,031 & ,049 & , 080 & ,641 &, 530 \\
\hline \multirow[t]{2}{*}{1} & REGR factr score & 2 for analysis 1 & ,317 & ,049 & 810 & 6,521 & ,000 \\
\hline & EWGR factor score & 3 for analysis 1 & 107 & ,049 & ,273 & 2,197 & ,042 \\
\hline
\end{tabular}

a. Variable dependiente: $Y$

Tabla 14. Coeficiente de correlación de SPEARMAN entre la competitividad y las variables independientes

\begin{tabular}{ccc}
\hline VARIABLES & r SPEARMAN & SIGNIFICANCIA \\
\hline$Y, X 1$ & 0.043 & 0.852 \\
$Y, X 2$ & 0.247 & 0.280 \\
$Y, X 3$ & 0.204 & 0.376 \\
$Y, X 4$ & 0.078 & 0.736 \\
$Y, X 5$ & -0.042 & 0.856 \\
$Y, X 6$ & -0.091 & 0.693 \\
$Y, X 7$ & 0.074 & 0.751 \\
$Y, X 8$ & -0.100 & 0.667 \\
$Y, X 9$ & 0.520 & 0.016 \\
$Y, X 10$ & 0.777 & 0.000 \\
$Y, X 11$ & 0.709 & 0.000 \\
\hline
\end{tabular}




\section{CONCLUSIONES}

Los factores que explican la variabilidad de la competitividad de los proyectos de servicios de extensión con $83.5 \%$ de probabilidad son: $\mathrm{F} 1=$ APORTES que integra a X1 (meses de duración del proyecto), X2 (aportes de INCAGRO) y X3 (alianza estratégica); F2 = TÉCNICO que agrupa X10 (servicios ofrecidos a clientes) y X11 (equipos técnicos comprometidos); $\mathrm{y}$ el factor $\mathrm{F} 3=$ IMPACTO que integra X4 (retorno económico) y X9 (impacto ambiental). E1 91\% de la variable aporte de la alianza estratégica (X3) es explicada por el modelo factorial hallado, mientras que la variable menos explicada por el modelo factorial es la variable X1 (meses) con sólo el 60.5\%.

Del análisis de regresión lineal múltiple se concluye que la variabilidad de la competitividad de los proyectos de extensión en estudio son explicadas en aproximadamente un $74 \%$ por la ecuación de regresión $\mathrm{Y}=3.429+0.031 \mathrm{~F} 1+0.317 \mathrm{~F} 2+0.107 \mathrm{~F} 3$; en donde el factor F2 TÉCNICO [X10 (servicios ofrecidos a clientes) y X11 (equipos técnicos comprometidos)] tiene el mas alto puntaje y por cada punto que este factor aumente, la competitividad aumentará en promedio en 0.317 , si se mantienen constante los puntajes de los otros factores. Con el factor F3 IMPACTO [X4 (retorno económico) y X9 (impacto ambiental)] se estima que por cada punto adicional de este factor se estima que la competitividad aumentará en promedio en 0.107, manteniéndose constante, los puntajes de los otros factores. Finalmente, por cada punto adicional en el puntaje del Factor F1 APORTES X1 [(meses de duración del proyecto), X2 (aportes de INCAGRO) y X3 (alianza estratégica)], se estima que la competitividad aumentará en promedio solamente 0.031 , manteniéndose constante, los puntajes de los otros factores.

Con el cálculo de los valores de los coeficientes de correlación no paramétrico de Spearman se concluye que la competitividad de los proyectos de servicios de extensión está significativamente correlacionada con las variables impacto ambiental (X9), servicios ofrecidos a los clientes (X10) y equipos técnicos (X11), es decir, la mejora de los valores de una de estas variables, obviando la presencia de las otras variables permitiría una mejora en el nivel de la competitividad. Los resultados también permiten concluir que la competitividad tiene un grado de asociación débil con las variables X2 (aporte INCAGRO), X3 (aporte alianza estratégica), X4 (retorno económico), X7 (eficacia), X1 (meses), X5 (beneficio costo INCAGRO), X6 (beneficio costo alianza estratégica) y X8 (eficiencia).

Todo lo anterior nos permite concluir que para mejorar la competitividad de los proyectos de Servicios de Extensión es imprescindible ofrecer buenos servicios a los clientes, contar con equipos técnicos de extensión comprometidos, buenos retornos económicos de las tecnologías ofrecidas y con bajo impacto ambiental. Las variables tiempo de duración de los proyectos, los aportes económicos, las alianzas estratégicas tienen un grado de asociación débil con la competitividad de los proyectos de extensión.

\section{BIBLIOGRAFÍA CITADA}

Benites, J. 2013. Hay una Oportunidad Única para el Agro. Agronoticias. 30 de noviembre 2013. Año XXXV, no. 394,p 25.

Benites, J; Wiener, H. 2008. INCAGRO: Converting ideas into values. (http://es.scribd.com/doc /24736660/INCAGRO-Converting-Ideas-IntoValues)

Janvry, A. 2009. La situación de la agricultura mundial y sus efectos en América Latina. En: Economía y sociedad. Lima: Consorcio de Investigación Económica y social (CIES):(71): 9 -13 .

Norton, R.D. 2004. Política de desarrollo agrícola. Conceptos y principios. Material conceptual y técnico. Capacitación en políticas agrícolas y alimentarias $\mathrm{N}^{\circ} 2$. Organización de las Naciones Unidas para la Agricultura y la Alimentación (FAO). Roma.

Ortiz, R. 2009. Evaluación de los servicios de extensión en Nicaragua: Managua: Organización de las Naciones Unidas (FAO). p. 4-5.

Recibido: 10/02/2014

Aceptado para publicación: 27/04/2014 
